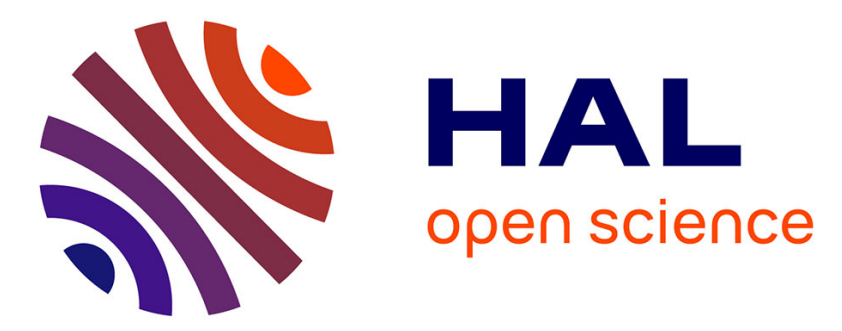

\title{
Second harmonic modulated photo-reflectance imaging of integrated circuits
}

\author{
Z.-L. Qian, S.-Y. Zhang, Y.-S. Lu, J. He, M. Tao
}

\section{To cite this version:}

Z.-L. Qian, S.-Y. Zhang, Y.-S. Lu, J. He, M. Tao. Second harmonic modulated photo-reflectance imaging of integrated circuits. Journal de Physique IV Proceedings, 1994, 04 (C7), pp.C7-203-C7-206. 10.1051/jp4:1994749 . jpa-00253278

\section{HAL Id: jpa-00253278 https://hal.science/jpa-00253278}

Submitted on 1 Jan 1994

HAL is a multi-disciplinary open access archive for the deposit and dissemination of scientific research documents, whether they are published or not. The documents may come from teaching and research institutions in France or abroad, or from public or private research centers.
L'archive ouverte pluridisciplinaire HAL, est destinée au dépôt et à la diffusion de documents scientifiques de niveau recherche, publiés ou non, émanant des établissements d'enseignement et de recherche français ou étrangers, des laboratoires publics ou privés. 


\title{
Second harmonic modulated photo-reflectance imaging of integrated circuits
}

Z.-L. Qian, S.-Y. Zhang, Y.-S. Lu, J. He and M. Tao

Institute of Acoustics and Lab. of Modern Acoustics, Nanjing University, Nanjing 210093, China

\begin{abstract}
The second harmonic imaging of the modulated photoreflectance (MPR) signals of integrated circuits has been obtained. The second harmonic imaging has special NDE capability for some surface and subsurface structures of semiconductor devices, particularly for the ion implantation process, based on the distinctive mechanism of the nonlinear MPR effect.
\end{abstract}

\section{INTRODUCTION}

In recent years, the nonlinear effects of modulated photoreflectance (MPR) in semiconductors have attracted great attention because it is related to the third order nonlinear optical susceptibility, the density of the surface energy state and the Auger recombination process [1-3]. Besides, it has been found that the 2nd harmonic MPR detection capability for the ion implantation is more sensitive than that of the fundamental, specially before the annealing of the wafers [1-2]. All of these indicate that the 2nd harmonic MPR signals may give some particular information on the surface and subsurface structures of semiconductor materials and devices.

In this paper, some results of the 2nd harmonic MPR imaging of IC devices are presented. Compared with the fundamental MPR imaging, the 2nd harmonic imaging has the distinctive NDE capability to detect the regions with the different ion-impanation processes and some subsurface structures because of the different mechanisms of the fundamental and the 2nd harmonic MPR effects. As pointed out in Ref [2], the 2nd harmonic MPR signal is only contributed by the plasma wave, while the fundamental MPR signal is due to the joint contributions of the plasma and thermal waves. In order to explain the difference between the 2 nd harmonic phase images and the fundamental ones, we calculate the phase sensitivities of the fundamental and the 2nd harmonic MPR signals changing with the bulk lifetime of the excess carriers in the different surface recombination velocities, which are in agreement with the experimental phenomena.

\section{EXPERIMENT}

Our experimental instruments are similar to those of Rosencwaig [4], in which the fundamental and second harmonic MPR signals are easily detected by the lock-in amplifier (EG\&G 5302). The pseudo-color MPR images at the fundamental (f) and the 2 nd harmonic ( $2 \mathrm{f}$ ) of the modulation frequency 
can be obtained simultaneously through scanning the sample. In order to detect the $2 \mathrm{f}$ MPR signal, the waveform of the modulated voltage driving the acousto-optic modulator is a square wave with $50 \%$ duty cycle, which should principally have no $2 \mathrm{f}$ component.

The integrated circuit (IC) sample is a MOS-structured ROM (Read Only Memory) device programmed by the ion implantation. The word lines are made of the polycrystal-Si and the bit lines are made of aluminum. The source and drain regions are formed after the annealing for the first high-dosed ion implantation. The logic state of every cell is determined by the second medium-dosed ion implantation in the gate region under the word line and thin oxide layer.

Fig.1(a) and (b) show the typical amplitude and phase images of the sample at the frequency $f$ about $270 \mathrm{kHz}$, respectively. The first and second ion-implanted regions are all shown in the amplitude image, but the phase image is less sensitive to these areas. Fig.1(c) and (d) are the amplitude and phase images at the 2nd harmonic frequency, respectively. Compared with Fig.1(a) and (b), we can see that the 2nd harmonic MPR amplitude image Fig.1(c) mainly reflects the first ion-implanted regions (sources and drains), while the phase image at $2 \mathrm{f}$ apparently reflects the second ion-implanted regions, i.e., displays the logic states, as shown in Fig.1(d), in which some light (brown) areas are surrounded by dark (blue) blocks which may indicate the "on" logic states and the others are "off" programmed by the second ion implantation.

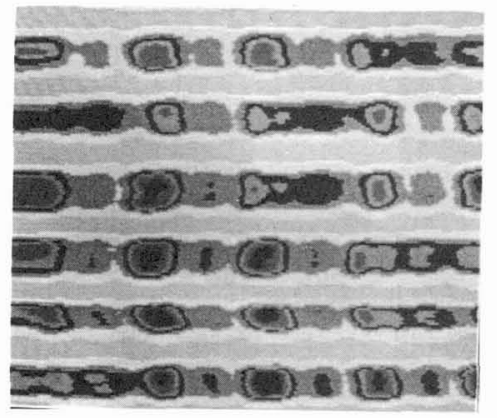

(a)

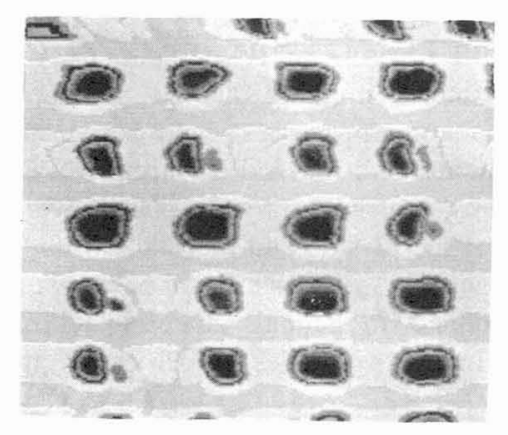

(c)

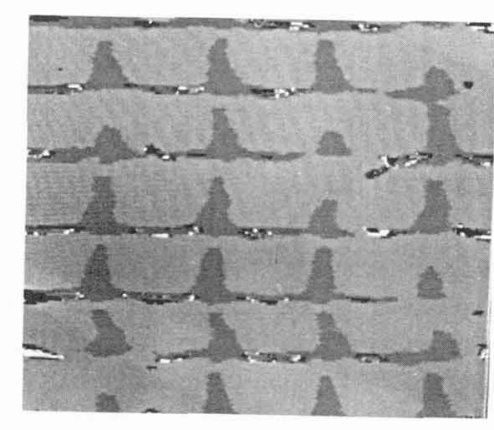

(b)

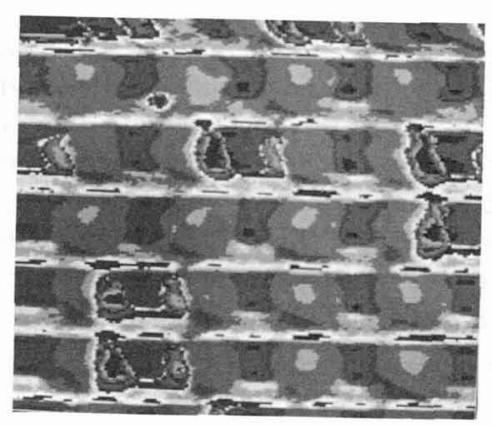

(d)

Fig. 1 The MPR images of a ROM programmed by the ion implantation (a)amplitude(f),(b)phase(f),(c)amplitude(2f),(d)phase(2f) 
In addition, we have also obtained several 2nd harmonic MPR images of some other IC devices. We find the 2nd harmonic MPR images are more sensitive to the structures on the surface and subsurface, which always can not be seen by the optical microscopy and the fundamental MPR images.

\section{DISCUSSIONS}

The mechanisms of nonlinear MPR effect in semiconductors have been studied by some authors[1-3]. They suggested that the photo-electro-reflectance (PER) effect and the Auger recombination effect are the main contributors to the nonlinear MPR signals of semiconductors. The former depends greatly on the surface energy state density and the third order nonlinear optical susceptibility of the semiconductor [1,2], and the latter depends strongly on the recombination centre density in the bulk and on the surface of the semiconductor [3]. Here, we mainly consider the gate regions with medium-dosed ion implantation under the illustration of the laser beam with sufficiently high intensity, thus we pay more attention to the PER effect but neglect the contribution of Auger recombination. Therefore, the 2nd harmonic MPR signal can be written as follows [2]:

$$
\frac{\Delta R}{R_{0}}(2 f)=H^{2} P_{1}(0)^{2} e^{2(2 \pi i f)}
$$

where

$$
H=\frac{e^{2}\left(\mu_{n}+\mu_{p}\right) N_{s} \chi^{(3)}}{2 \pi n\left(n^{2}-1\right) \epsilon \sigma}
$$

and $P_{1}(0)$ is the fundamental frequency plasma wave in the surface of the sample, $e$ is the electron charge, $\mu_{\mathrm{v}}\left(\mu_{\mathrm{p}}\right)$ is the mobility of the electron (hole), $\mathrm{N}_{\mathrm{s}}$ is the density of the surface energy state, $\chi^{(3)}$ is the third order nonlinear optical susceptibility, $\mathrm{n}$ is the optical refractive index, $\epsilon$ is the dielectric function, and $\sigma$ is the electric conductivity.

After the ion implantation, the lattice defects are produced in the surface and bulk of the semiconductor wafer. Although the implanted wafer is always annealed, some residual defects still exist in the surface and the bulk. The residual defects and the electrically activated impurities will change the thermal and electrical properties such as the thermal conductivity, the density of the surface energy state and the lifetime of excess carriers. According to the theory above, the 2nd harmonic MPR signal is only due to the contribution of the plasma wave, while the fundamental MPR signal is the joint contributions of the plasma and thermal waves at the modulation frequency of our experiment [5]. Therefore, the fundamental MPR amplitude image displays the changes of the electrical and thermal properties, and the 2nd harmonic MPR amplitude image mainly detects the change of the electrical properties, which is probably the reason why there exist some differences between Fig.1(a) and (c), as well as between Fig. 1(b) and (d).

We can see that, the second harmonic MPR phase image reflects the second ion-implanted regions connected with the logic states. It is evident that, according to Eq.(1), the change of $\chi^{(3)}$ and $\mathrm{N}_{\mathrm{s}}$ can not lead to the change of the $2 f$ MPR phase. Thus, the change of the $2 \mathrm{f}$ MPR phase results from the change of $P_{1}(0)$, which varies with the electrical properties, such as the bulk lifetime, the surface and interface recombination velocities of the excess carriers. Therefore, the 2 nd harmonic MPR phase image can represent the changes of the electrical properties since it only reflects the contribution of the plasma wave. On the other hand, because the phase difference between the contributions of the thermal and plasma waves is larger than $90^{\circ}$, the fundamental MPR can not sensitively reflects the changes of the bulk lifetime and the surface and interface recombination velocities of the excess carriers. Thus, the 
increase of the 2nd MPR phase signal of the gate regions in Fig.1(d) comes from the variations of the bulk lifetime and/or the surface and interface recombination velocities of the carriers induced by the programmed ion implantation.

As an example, we calculate the dependence of the phase sensitivities $(\mathrm{d} \theta / \mathrm{d}(\ln (\tau))$ of the fundamental and the 2nd harmonic signals on the bulk lifetime $(\tau)$ of excess carriers in different surface recombination velocities $\left(S_{f}\right)$ as shown in Fig. 2. It is clear that the 2f MPR phase is more sensitive to the changes of the bulk lifetime and the surface recombination velocity of the excess carriers at appropriate operating frequencies.

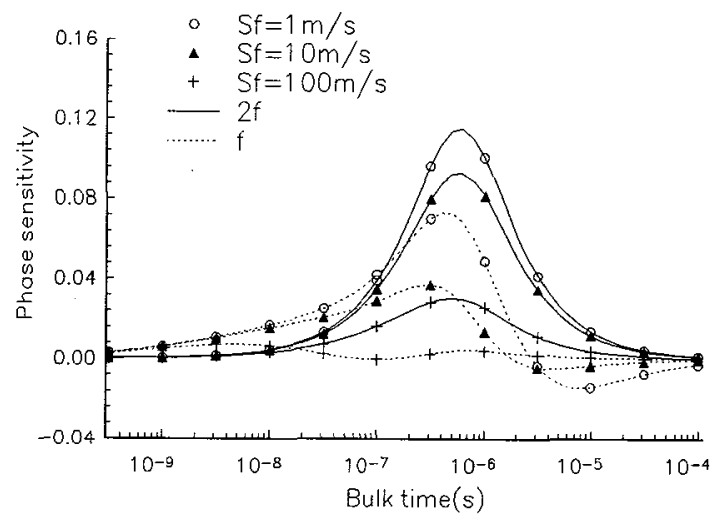

Fig.2. The dependence of the phase sensitivities of MPR signal on the bulk lifetime

\section{CONCLUSION}

The 2nd harmonic MPR images are obtained for a MOS-structured ROM device. It is manifested that the 2nd harmonic MPR imaging has its own special NDE capability. Specially, the 2nd harmonic MPR phase image is more sensitive than the fundamental MPR phase for the ion-implanted gate regions with medium dose. Thus it can be used to detect the changes of the bulk lifetime and the surface and interface recombination velocities of the excess carriers sensitively, as well as to detect the subsurface structures successfully. The differences between the 2nd harmonic MPR images and the fundamental MPR images can primarily be interpreted by the corresponding theoretical mechanisms, i.e., the fundamental MPR signal results from both thermal and plasma waves, but the 2nd harmonic MPR signal comes only from the plasma wave.

\section{REFERENCES}

[1] S.Y. Zhang, Y.S. Lu and H.S. Li, in Photoacoustic and Photothermal Phenomena III, Ed., D.Bicanic, (Springer-Verlag ,1992) pp.369-371.

[2] Y.S. Lu, S.Y. Zhang and Z.L. Qian, J. Appl. Phys. 74 (1993) 4710-4715.

[3] B.C. Forget, D. Fournier and V.E. Gusev, Appl. Phys. Lett. 61 (1992) 2341-2343.

[4] A.Rosencwaig, Photoacoustic and Thermal Wave Phenomena in Semiconductors, Ed., A. Mandelis, (Elsvier Science, 1987) pp.97-135.

[5] S.Y. Zhang, Z.L. Qian, Y.S. Lu, J. He and Z.N. Zhang, "Ion implantation in semiconductor wafers studied by photothermal technique", 7th Asian-Pacific Conf. on NDT, Ed., W.L Luo, (Shanghai, China, 1993) pp.850-855. 